activities that don't technically require IACUC coverage.

We have experienced several advantages with the use of this form. First, it promotes forethought in club advisors when planning an animal-based activity or demonstration, hopefully preventing or minimizing on-the-fly decisions that may not be in the best interest of the animal or the university. Second, the form helps the IACUC to be aware of such activities happening on campus, which supports positive relationships between clubs and the IACUC. Third, should a welfare concern arise related to club activities, the IACUC already has a reference point from which to start reviewing the concern.
Maggie Behnke* and Sally Olson

Comparative Medicine Group,

Kansas State University, Manhattan,

KS, USA.

${ }^{*}$ e-mail:msbehnke@ksu.edu

Published online: 19 February 2019

https://doi.org/10.1038/s41684-019-0240-y

\title{
What's the big deal?
}

\begin{abstract}
$\mathrm{n}$ the United States Animal Welfare Act, $\$ 2132$ Definitions, (g), “The term 'animal' means any live or dead dog, .....as the Secretary may determine is being used, or is intended for use, for research, testing, experimentation, or exhibition purposes, or as a pet; ..." (emphasis added). Thus, one could make a good case that Great Eastern University's IACUC approval is needed for the specified activities. IACUC approval would also be justified given the stated facts that the students and faculty advisor are associated with the university and, most importantly, that the activities involving the dogs are occurring on university property,
\end{abstract}

which at least implies university approval of the content of the activities.

All of these facts aside, what would be the harm of asking for IACUC approval? As described, the CAFE club's activities are unlikely to be controversial, nor would there seem to be any significant animal welfare issues since all of the dogs are owned by the participants; thus, a wellwritten protocol should have little problem being approved.

Finally, although the risk may be slim, there is always the possibility that one of the participants could be injured by an animal bite or physical trauma from mishandling of the dogs. IACUC approval of a well-written protocol that includes adequate preventive procedures and "Hold harmless" agreements to be provided by participants could possibly mitigate the university's liability in such an event.

\section{Richard C. Simmonds ${ }^{1,2}$}

${ }^{1}$ Nevada System of Higher Education, Reno, NV, USA. ${ }^{2}$ University of Nevada, School of Medicine, Reno, NV, USA.

e-mail: rsimmonds@unr.edu

Published online: 19 February 2019

https://doi.org/10.1038/s41684-019-0241-x

\section{Regulations, funding and institutional responsibility}

W hether or not IACUC approval is required for the CAFE club's activities depends on several factors. The first consideration is whether the animals are under the auspices of the Animal Welfare Act Regulations (AWAR) and/or Public Health Service (PHS) policy. The pet animals used by the CAFE club to teach members how to groom, train, and exhibit animals in show are not owned by the university. Therefore, the regulations do not apply and IACUC approval is not required. Per PHS Policy, the requirement for IACUC approval of the CAFE club's activities depends on whether Great Eastern University has an Animal Welfare Assurance and voluntarily includes all animals under their Assurance. If they do, then IACUC approval would be required ${ }^{1-4}$.

A second consideration involves how the CAFE club and its activities are funded.
If funding is provided by a private agency, there are no federal or state laws that require the agency to obtain IACUC approval of animal use projects if the animals are not AWAR-regulated covered species. Nonetheless, private or public agencies may specifically require IACUC approval prior to release of funds. If the university has an Animal Welfare Assurance that commits the institution to comply with the PHS Policy for all animals, IACUC approval would be required regardless of funding source ${ }^{1,2}$.

A final consideration is the university's ethical responsibility for the use of animals by the CAFE club. If there are no regulatory or funding agency requirements for IACUC approval, the university should have a mechanism in place to address all animal related activities on it campus, such as a written policy that covers the use of pets or other privately owned animals used for CAFE club's activities ${ }^{2}$.

Joanne Tetens-Woodring ${ }^{\star}$, David A. Custer and Nathalie Conway-James

University of Cincinnati, Cincinnati, OH, USA.

*e-mail: tetensje@ucmail.uc.edu

Published online: 19 February 2019

https://doi.org/10.1038/s41684-019-0242-9

References

1. Brown, M. \& Shepherd, M. J. Circumstances Requiring an IACUC. The IACUC Handbook 3rd edn (CRC Press, Boca Raton, Florida, 2014).

2. Laber, K. \& Smith, A. Submission and Maintenance of IACUC Protocols. The IACUC Handbook 3rd edn (CRC Press, Boca Raton, Florida, 2014).

3. Animal Welfare Act and Animal Welfare Regulations. (Animal and Plant Health Inspection Service APHIS 41-35-076, 2017).

4. Office of Laboratory Animal Welfare, National Institutes of Health. Public Health Service Policy on Humane Care and Use of Laboratory Animals (US Department of Health and Human Services, Bethesda, Maryland, USA, 2015). 